\title{
CREENCIAS DE LOS ESTUDIANTES DEL MÁSTER DE SECUNDARIA EN ESPAÑA-PORTUGAL
}

María-Pilar Molina-Torres

I Universidad de Córdoba (UCO), Córdoba, España; pilar.molina@uco.es

\section{Resumen}

El objetivo general de este estudio es analizar la realidad curricular y las opiniones de los estudiantes acerca de los métodos de enseñanza que conocen durante su formación profesional en el Máster de Profesorado de Educación Secundaria de la Universidad de Córdoba (España) y la Universidad de Oporto (Portugal). La metodología implementada nos ha permitido identificar mediante un cuestionario tipo encuesta las concepciones del alumnado sobre las metodologías y las estrategias didácticas que piensan serían más adecuadas para su futura práctica profesional y formación docente. Los resultados de la investigación señalan que los estudiantes desconocen los modelos didácticos a emplear en sus clases. Por último, este trabajo supuso una oportunidad para sondear las creencias que el alumnado tiene acerca de la excesiva teorización de las materias.

FORMACIÓN DE PROFESORES • EDUCACIÓN SUPERIOR • DIDÁCTICA • MÉTODOS DE ENSEÑANZA

\section{BELIEFS OF THE STUDENTS OF THE SECONDARY MASTER IN SPAIN-PORTUGAL Abstract}

The general objective of this study is to analyze the curricular reality and the opinions of the students about the teaching methods they know during their professional training in the Master's Degree in Secondary Education at the University of Córdoba (Spain) and the University of Porto (Portugal). The implemented methodology has allowed us to identify, through a questionnaire type survey, the students' conceptions of the methodologies and didactic strategies that they think would be more appropriate for their future professional practice and teacher training. The results of the investigation indicate that the students are unaware of the didactic models to be used in their classes. Finally, this work was an opportunity to probe the beliefs that students have about the excessive theorizing of subjects.

TEACHER EDUCATION • HIGHER EDUCATION • DIDACTICS • TEACHING METHODS 


\section{CRENÇAS DOS ALUNOS DO MESTRADO EM ENSINO SECUNDÁRIO EM ESPANHA-PORTUGAL}

\section{Resumo}

O objetivo geral deste estudo é analisar a realidade curricular e as opiniões dos alunos sobre os métodos de ensino que conhecem durante sua formação profissional no Mestrado em Ensino Secundário da Universidade de Córdoba (Espanha) e da Universidade do Porto (Portugal). A metodologia implementada nos permitiu identificar, através de uma pesquisa do tipo questionário, as concepções dos alunos sobre as metodologias e estratégias didáticas que consideram que seriam mais apropriadas para sua futura prática profissional e formação docente. Os resultados da pesquisa indicam que os alunos desconhecem os modelos didáticos a serem utilizados em suas aulas. Por fim, este trabalho representou uma oportunidade para sondar as crenças dos sobre a teorização excessiva das disciplinas.

FORMAÇÃO DE PROFESSORES • ENSINO SUPERIOR • DIDÁTICA • MÉTODOS DE ENSINO

\section{CROYANCES DES ÉTUDIANTS DE MAITRISE EN ENSEIGNEMENT SECONDAIRE EN ESPAGNE ET AU PORTUGAL}

\section{Résumé}

L'objectif général de cette étude est d'analyser la réalité du programme scolaire et les opinions des étudiants sur les méthodes d'enseignement auxquelles ils sont exposés tout au long de leur formation professionnelle dans le cadre du cours de maîtrise en Enseignement Secondaire à l'Université de Cordoue (Espagne) et à l'Université de Porto (Portugal). La méthodologie mise en œuvre a permis d'identifier, à travers une enquête par questionnaire, les conceptions des étudiants sur les méthodologies et les stratégies didactiques qu'ils jugent les plus adaptées à leur future pratique professionnelle ainsi quà la formation des enseignants. Les résultats de la recherche montrent que les étudiants ne connaissent pas les modèles didactiques qu'ils devront utiliser dans leurs propres classes. Par ailleurs, ce travail a permis d'examiner les croyances des étudiants quant à la théorisation excessive des disciplines. 


\section{Presentación y justificación del estudio}

Las creencias de los estudiantes del Máster de Formación del Profesorado de Enseñanza Secundaria (MAES) aportan una visión decisiva para dar sentido a las problemáticas metodológicas y a la enseñanza de la historia. En esta dirección, el término "creencias" se ciñe a las experiencias previas que el alumnado ha ido incorporando a su cultura pedagógica y sobre todo a la implicación que han tenido con las titulaciones e itinerarios formativos de los que proceden. Para su justificación, la recopilación y el análisis de dichas creencias acerca de los procesos de enseñanza y la Didáctica de la Historia nos sitúan en el complicado proceso de la formación inicial del profesorado novel.

En este ámbito, asumen una importancia significativa estas concepciones que refuerzan la incorporación de metodologías activas de aprendizaje a nuestra práctica docente para mejorar la formación didáctica y las competencias profesionales del alumnado (Domínguez, 2015). En este proceso de enseñanza profesional es necesario profundizar en los aprendizajes experienciales asociados a los acontecimientos históricos que conocen y también sopesar si los conocimientos conceptuales y memorísticos permiten una actitud reflexiva que estimule la problematización de contextos en los que trabaja un docente (Montanares \& Llancavil, 2016).

Estos conceptos temporales se convierten en elementos esenciales para interpretar qué saben y cómo quieren conocer los estudiantes el tiempo histórico (Santisteban, 2007). La perspectiva histórica implicaría una contextualización temporal-histórica que sitúe al alumnado en un hecho concreto del pasado. Como apunta Seixas (2011), el significado histórico no es inseparable del pasado en sí mismo, sino que se crea a partir de la relación entre el historiador y el pasado. De este modo, los estudiantes se benefician y aprenden de la inclusión de historias en sus aulas de secundaria, lo que facilita su participación en un pensamiento histórico complejo sobre temas que pueden tener un gran peso emocional (Levy, 2017). Por su parte, Barca (2010) piensa que la narrativa histórica manifiesta los paralelismos que su narrador encuentra entre el pasado, el presente y ocasionalmente el futuro, desde un punto de vista individual y social.

Se trata, en definitiva, de interpretar el pasado y construir ese pensamiento histórico siguiendo los métodos del historiador para aprender las herramientas suficientes en la práctica profesional de los estudiantes de posgrado (Vansledright, 2014). El pensar históricamente no se ciñe a recordar los hechos y acontecimientos del pasado (Gómez Carrasco et al., 2014). Ese conocimiento del pasado y su comprensión histórica implicarían la puesta en práctica de habilidades de pensamiento alejadas de la memorización conceptual (Sáiz Serrano \& Gómez Carrasco, 2016). De ahí que, desde el punto de vista de la investigación narrativa y la memoria social, exista una estrecha relación entre las áreas de Ciencias Sociales y Humanidades (Liu, 2013).

Indudablemente en la última década la investigación en la Didáctica de la Historia ha aportado un significativo número de trabajos acerca de la educación histórica del alumnado (Chapman, 2011; Gairín, 2011; Gómez Carrasco \& Miralles Martínez, 2015; Hernández Amoros \& Carrasco Embuena, 2012; Ibáñez-Etxeberria et al., 2015; Levstik, 2016; Prats \& Santacana, 2011; Rivero \& Souto, 2019; Rüsen, 2005; Schmidt \& Barca, 2009; Seixas \& Morton, 2013). Estos estudios configuran una línea de trabajo que se ha tratado de forma parcial sin profundizar en aspectos tan significativos como la construcción de la identidad profesional del profesorado de secundaria y el desarrollo de las competencias para la práctica docente. De hecho, se espera que los docentes tengan unos conocimientos y actitudes implícitos en su profesión (Martínez de la Hidalga \& Villardón-Gallego, 2015) y prioricen las competencias educativas sobre los contenidos teóricos (Blömeke et al., 2016). Para Serrano y Pontes (2016), en este desarrollo profesional es imprescindible la motivación del alumnado para implicarse en el desempeño de sus tareas competenciales. 
En este contexto, es evidente que la educación histórica, en un contexto formal, permita al alumnado de secundaria actuar y reflexionar sobre las herramientas educativas que utilizarán en sus enseñanzas. Como indica Marques (2006) es necesario asegurar la comprensión del conocimiento (científico) dentro de un marco de comprensión que no renuncie a la temporalidad, la espacialidad y la contextualización de los datos, de forma que podamos confirmar que el estudiante puede comunicar "históricamente" lo aprendido, de la manera más adecuada. Al respecto Jiménez et al. (2018) apuntan que la formación didáctica del profesorado en los métodos de enseñanza es una oportunidad para el aprendizaje del docente y una mayor implementación de éstos en su desarrollo profesional.

Para ello, la formación previa del profesorado de historia tendría que mejorar desde sus niveles básicos y orientarla al ejercicio profesional, lo que favorece la educación histórica de su futuro alumnado (Sáiz Serrano \& López-Facal, 2015). De hecho, la relación entre la formación inicial y el acceso a la función docente es un problema de gran importancia tanto para los futuros profesores como para el sistema educativo y para la propia sociedad (Lorenzo et al., 2015). También, es necesario que la formación inicial de los docentes vincule esa educación histórica con la realidad universitaria (Trigueros Cano et al., 2018) y mejore el aprendizaje mediante la relación entre tutores universitarios y profesores de secundaria. Por su parte, Nóvoa (2009) nos habla de tres fases de formación para los profesores de enseñanza secundaria: la licenciatura universitaria, el máster de educación y un periodo inicial en la profesionalización docente.

Ahora bien, la formación por competencias del profesorado debe facilitar las situaciones reales para experimentar un enfoque menos teórico de las disciplinas históricas (Estepa \& Martín, 2018). También es necesario atender a la demanda de cambios de ritmo de trabajo y considerar la innovación docente como un elemento imprescindible de nuestro proceso de formación. Por su parte, Krichesky y Murillo (2018) afirman que no todas las prácticas colaborativas estimulan mejoras sustanciales en la enseñanza o desarrollan la capacidad de innovación del profesorado. Lo que, en gran medida, confirma que la colaboración como estrategia en la práctica educativa refuerza la autonomía del docente y sus habilidades pedagógicas (Imbernón et al., 2020).

En este sentido, resulta evidente que el aprendizaje de la historia no debe limitarse solamente a memorizar y reproducir contenidos históricos o narrativas basadas en el pasado (Sáiz Serrano \& Fuster Garcia, 2014). Al respecto Marques (2016) advierte que debido a que la historia, como conocimiento del pasado, es un proceso de construcción de una narrativa de ese pasado, necesita un trabajo de aproximación entre la sociedad de ayer y de hoy. Desde esta perspectiva, todo apunta a que la formación teórica y práctica relacionada con la profesión docente sería un trabajo de coordinación entre los docentes universitarios y de educación secundaria (Imbernón, 2019). Es más, la enseñanza de la historia a lo largo de las etapas básicas debería combinar el aprendizaje de conocimientos e información histórica con el de destrezas o competencias históricas, derivados del trabajo del historiador. Es preciso considerar cómo la formación del pensamiento histórico en las aulas forja el interés de los alumnos por la historia y el desarrollo del pensamiento crítico (Gómez Carrasco et al., 2014).

Por otro lado, es preciso señalar que el proceso formativo de los estudiantes del MAES de la Universidad de Córdoba se verifica con una titulación universitaria que corresponda con la especialidad elegida: Ciencias Sociales, Geografía e Historia. En cambio, para realizar el Mestrado em Ensino de História no $3^{\circ}$ ciclo do Ensino Básico e no Ensino Secundário en la Universidad de Oporto la titulación de acceso sería la Licenciatura de Historia. De este modo, los contenidos y las competencias adquiridos en sus grados o licenciaturas se reforzarán curricularmente con sus estudios de posgrado, en los que tendrán que cursar las siguientes materias y créditos (Cuadro 1): 
CUADRO 1

ASIGNATURAS Y CRÉDITOS DE LA U.PORTO Y DE LA UCO

\begin{tabular}{|c|c|c|c|}
\hline \multicolumn{2}{|c|}{ FLUP (Universidad de Oporto) } & \multicolumn{2}{|l|}{ FCCEE (Universidad de Córdoba) } \\
\hline Asignaturas & Créditos & Asignaturas & Créditos \\
\hline Políticas Educativas e Curriculo & 6 & Aprendizaje y desarrollo de la personalidad & 4 \\
\hline Didática da História I & 6 & Procesos y contextos educativos & 4 \\
\hline História da Cultura e das Artes & 6 & Sociedad, Familia y Educación & 4 \\
\hline Psicologia da Educação & 6 & Gestión de la Convivencia Escolar & 4 \\
\hline História Económica & 6 & $\begin{array}{l}\text { Las TIC como recursos educativos para la } \\
\text { enseñanza secundaria }\end{array}$ & 4 \\
\hline Ética e Educação & 6 & Plataformas de enseñanza virtual & 4 \\
\hline Didática da História II & 6 & Técnicas de comunicación oral en la docencia & 4 \\
\hline Aplicações Didáticas em História & 6 & $\begin{array}{c}\text { Aprendizaje y enseñanza de las materias } \\
\text { correspondientes en Ciencias Sociales, Geografía } \\
\text { e Historia }\end{array}$ & 12 \\
\hline Investigação Educacional & 6 & $\begin{array}{c}\text { Complementos para la formación disciplinar en } \\
\text { Ciencias Sociales: } \\
\text { Geografía e Historia }\end{array}$ & 6 \\
\hline A Web e o Ensino da História & 6 & $\begin{array}{c}\text { Innovación docente e iniciación a la investigación } \\
\text { educativa en Ciencias Sociales: Geografía e } \\
\text { Historia }\end{array}$ & 6 \\
\hline Iniciação à Prática Profissional & 48 & Prácticas externas & 10 \\
\hline Perspetivas em Educação Histórica & 6 & Trabajo Fin de Máster & 6 \\
\hline Problemáticas Históricas & 6 & & \\
\hline
\end{tabular}

Fuente: Elaboración propia.

Estas materias deben afianzar la formación inicial que incluya elementos curriculares, contenidos teóricos de la especialidad, estrategias de aprendizaje, adquisición de competencias, gestión de los centros educativos, métodos de enseñanza de la historia, todos ellos necesarios para construir su desarrollo profesional. Para el caso concreto de la Facultad de Letras de la Universidad de Oporto, Marques y Pinto (2018) indican que: "la intención del máster fue desarrollar un marco conceptual y familiarizarse con varios procedimientos que permiten una autonomía progresiva del conocimiento de la enseñanza en el aprender a hacer del profesor" (p. 18). En cierto modo, como apunta López-Facal (2012) los complementos siempre deben estar referidos a las materias de secundaria propias de la especialidad docente, no de la especialidad académica universitaria.

Por todo ello, el objetivo prioritario de este estudio se ha centrado en detectar las creencias que los estudiantes del Máster de Profesorado en Enseñanza Secundaria de las universidades de Oporto (Portugal) y Córdoba (España) tienen en relación con las metodologías de aprendizaje que consideran más adecuadas para implementarlas en su futuro profesional. Para alcanzar este objetivo se recogen los siguientes objetivos específicos: (1) conocer los métodos de enseñanza recibidos en su formación universitaria; (2) identificar su interés en relación con los conocimientos del pasado y su interpretación; (3) indagar las dificultades académicas que tienen para evaluar los contenidos históricos; y (4) analizar los recursos didácticos para implementar una metodología histórico-educativa. 


\section{Metodología}

\section{Participantes y muestra}

La muestra de estudio estuvo compuesta por estudiantes del Máster de Profesorado en Enseñanza Secundaria de la Universidad de Córdoba $(\mathrm{N}=118)$ que cursaban la asignatura de "Aprendizaje y enseñanza de las asignaturas correspondientes en Ciencias Sociales: Geografía e Historia”, y de la Universidad de Oporto $(\mathrm{N}=26)$ que cursaron la asignatura de "Didáctica de la Historia I". La edad media del alumnado que participó en esta investigación es de 24 años.

Para la selección de esta muestra que no tuvo un carácter probabilístico se seleccionó a aquellos grupos de participantes en los que los docentes responsables de las asignaturas se habían implicado de forma directa en la investigación educativa. Aunque el número de estudiantes portugueses es inferior, la representatividad de la muestra es semejante. Asimismo, la elección de los dos países se debe a las diferencias curriculares y competenciales en la enseñanza de la historia.

\section{Diseño de la investigación}

En este estudio se ha empleado un cuestionario cuantitativo no experimental, tomando como ejemplo los trabajos de Hernández-Pina y Maquilón (2010). Este enfoque de investigación permitió aproximarnos a las creencias de los estudiantes en cuanto a los estilos de enseñanza más apropiados para adquirir conocimientos de historia, además de a las diferentes perspectivas que tienen sobre la educación y el pensamiento histórico (Metzger \& Harris, 2018). Partiendo de estas premisas, con el análisis de este estudio pudimos comprender los fundamentos teóricos y prácticos en los que se sustenta la educación histórica.

Para ello, se diseñó una batería de preguntas de carácter cerrado, según las propuestas de Kvale (2011) y Moriña (2017), para analizar la capacitación metodológica y profesionalizante que el alu mnado adquiere en las sesiones teórico-prácticas de ambos másteres. Este tipo de estudios son habituales en las investigaciones relacionadas con el ámbito de la educación, ya que darían respuesta a la cantidad de los aprendizajes adquiridos y a la evaluación de las diferentes variables (García \& Cabero, 2011).

\section{Instrumento de recogida y análisis de los datos}

Los datos analizados forman parte de un cuestionario denominado "Creencias del profesorado en formación sobre los métodos de enseñanza de la Historia”. Este instrumento se centró en conocer los métodos de enseñanza recibidos en sus clases de historia en el Máster de Profesorado. Para este estudio se utilizó una escala tipo Likert de cinco valores que abarcan desde 1 que equivalía a totalmente en desacuerdo y 5 a totalmente de acuerdo. En la clasificación y elaboración de los 20 ítems del cuestionario se tuvo en cuenta el trabajo de Meschede et al. (2017). En su validación intervinieron cinco expertos en Educación Secundaria y Didáctica de la Historia de universidades españolas y portuguesas. Tras la aprobación de su estructura y contenidos por los expertos, los ítems fueron traducidos al portugués para tomar la muestra de la Facultad de Letras de la Universidad de Oporto. 


\section{CUADRO 2 \\ CUESTIONARIO “CREENCIAS DEL PROFESORADO EN FORMACIÓN SOBRE LOS MÉTODOS DE ENSEÑANZA DE LA HISTORIA"}

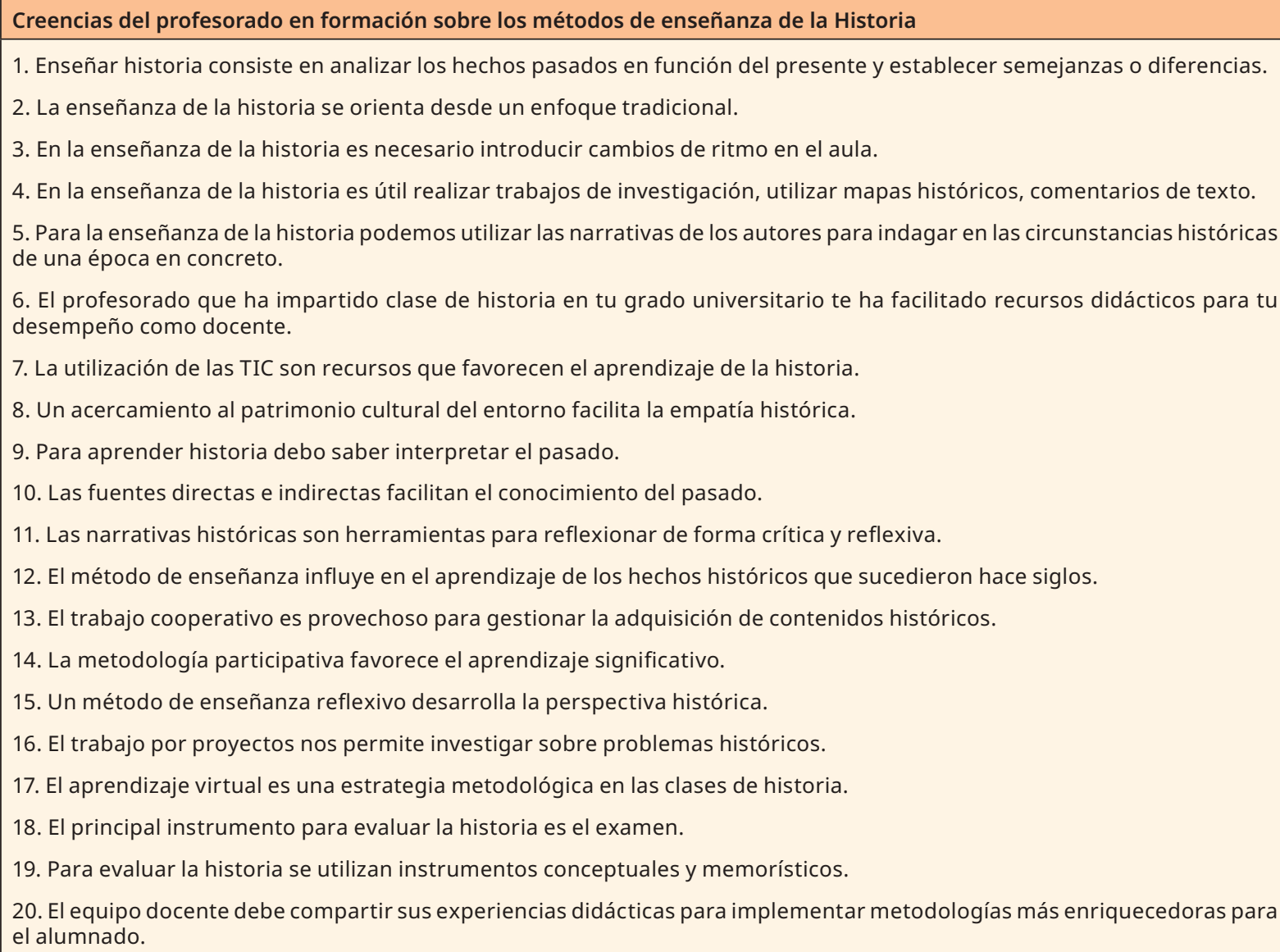

20. El equipo docente debe compartir sus experiencias didácticas para implementar metodologías más enriquecedoras para el alumnado.

Fuente: Elaboración propia.

Para dar respuesta a los objetivos planteados en el cuestionario se midieron frecuencias y porcentajes. En el análisis e interpretación de la información se utilizó como soporte técnico una hoja de cálculo Excel para realizar los gráficos y los cálculos que se requerían.

\section{Resultados}

Los resultados de las tablas que se analizan a continuación se han dividido en las dos muestras que representan a las universidades de España y Portugal, con el fin de analizar las valoraciones de ambos grupos de estudiantes y dar respuesta a los objetivos planteados al comienzo del estudio. Para concretar el primer objetivo centrado en conocer los métodos de enseñanza recibidos en la universidad, hemos tomado como referencia los ítems 2 y 14. Los resultados de la Tabla 1 muestran que la enseñanza universitaria favorece métodos de aprendizaje con un enfoque tradicional tanto en la Universidad de Oporto (84.6\%) como en la Universidad de Córdoba (85.6\%). Asimismo, los estudiantes de ambas universidades están totalmente de acuerdo con un $69.2 \%$ y un $74.6 \%$ en que la metodología participativa beneficia la adquisición de un aprendizaje significativo. 
TABLA 1

\section{MÉTODOS DE ENSEÑANZA IMPLEMENTADOS EN LA UNIVERSIDAD}

\begin{tabular}{|c|c|c|c|c|c|c|}
\hline \multicolumn{2}{|c|}{ Portugal } & $\begin{array}{l}\text { Totalmente en } \\
\text { desacuerdo }\end{array}$ & En desacuerdo & $\begin{array}{l}\text { Ni de acuerdo, } \\
\text { ni en desacuerdo }\end{array}$ & De acuerdo & $\begin{array}{l}\text { Totalmente de } \\
\text { acuerdo }\end{array}$ \\
\hline \multicolumn{7}{|c|}{ 2. La enseñanza de la historia en la universidad se orienta desde un enfoque tradicional } \\
\hline$\%$ & 100 & 0 & 0 & 11.5 & 3.9 & 84.6 \\
\hline Frq & 26 & 0 & 0 & 3 & 1 & 22 \\
\hline \multicolumn{2}{|c|}{ Portugal } & $\begin{array}{l}\text { Totalmente en } \\
\text { desacuerdo }\end{array}$ & En desacuerdo & $\begin{array}{l}\text { Ni de acuerdo, } \\
\text { ni en desacuerdo }\end{array}$ & De acuerdo & $\begin{array}{l}\text { Totalmente de } \\
\text { acuerdo }\end{array}$ \\
\hline \multicolumn{7}{|c|}{ 14. La metodología participativa favorece el aprendizaje significativo } \\
\hline$\%$ & 100 & 3.9 & 7.7 & 0 & 19.2 & 69.2 \\
\hline Frq & 26 & 1 & 2 & 0 & 5 & 18 \\
\hline \multicolumn{2}{|c|}{ España } & $\begin{array}{l}\text { Totalmente en } \\
\text { desacuerdo }\end{array}$ & En desacuerdo & $\begin{array}{l}\text { Ni de acuerdo, } \\
\text { ni en desacuerdo }\end{array}$ & De acuerdo & $\begin{array}{c}\text { Totalmente de } \\
\text { acuerdo }\end{array}$ \\
\hline \multicolumn{7}{|c|}{ 2. La enseñanza de la historia en la universidad se orienta desde un enfoque tradicional } \\
\hline$\%$ & 100 & 1.7 & 2.5 & 3.4 & 6.8 & 85.6 \\
\hline Frq & 118 & 2 & 3 & 4 & 8 & 101 \\
\hline \multicolumn{2}{|c|}{ España } & $\begin{array}{l}\text { Totalmente en } \\
\text { desacuerdo }\end{array}$ & En desacuerdo & $\begin{array}{l}\text { Ni de acuerdo, } \\
\text { ni en desacuerdo }\end{array}$ & De acuerdo & $\begin{array}{l}\text { Totalmente de } \\
\text { acuerdo }\end{array}$ \\
\hline \multicolumn{7}{|c|}{ 14. La metodología participativa favorece el aprendizaje significativo } \\
\hline$\%$ & 100 & 2.5 & 5.9 & 6.8 & 10.2 & 74.6 \\
\hline Frq & 118 & 3 & 7 & 8 & 12 & 88 \\
\hline
\end{tabular}

Fuente: Elaboración propia.

A continuación, para dar respuesta al segundo objetivo se han seleccionado los ítems 9 y 10. El primero muestra cierta incredulidad entre el alumnado portugués (42.3\%) para interpretar el pasado a través del conocimiento de la historia, mientras que el porcentaje de los estudiantes españoles es más elevado, 60.2\%. Para el ítem 10, relacionado con la utilidad de las fuentes directas e indirectas en el conocimiento del pasado, los porcentajes fueron discretos con un $23.1 \%$ para Portugal y el $44.9 \%$ para España.

TABLA 2

CONOCIMIENTO E INTERPRETACIÓN DEL PASADO HISTÓRICO

\begin{tabular}{|c|c|c|c|c|c|c|}
\hline \multicolumn{2}{|c|}{ Portugal } & $\begin{array}{c}\text { Totalmente en } \\
\text { desacuerdo }\end{array}$ & En desacuerdo & $\begin{array}{l}\text { Ni de acuerdo, } \\
\text { ni en desacuerdo }\end{array}$ & De acuerdo & $\begin{array}{l}\text { Totalmente de } \\
\text { acuerdo }\end{array}$ \\
\hline \multicolumn{7}{|c|}{ 9. Para aprender historia debo saber interpretar el pasado. } \\
\hline$\%$ & 100 & 11.5 & 7.7 & 11.5 & 27 & 42.3 \\
\hline Frq & 26 & 3 & 2 & 3 & 7 & 11 \\
\hline \multicolumn{2}{|c|}{ Portugal } & $\begin{array}{c}\text { Totalmente en } \\
\text { desacuerdo }\end{array}$ & En desacuerdo & $\begin{array}{c}\text { Ni de acuerdo, } \\
\text { ni en desacuerdo }\end{array}$ & De acuerdo & $\begin{array}{l}\text { Totalmente de } \\
\text { acuerdo }\end{array}$ \\
\hline \multicolumn{7}{|c|}{ 10. Las fuentes directas e indirectas facilitan el conocimiento del pasado. } \\
\hline$\%$ & 100 & 19.2 & 3.9 & 15.4 & 38.4 & 23.1 \\
\hline Frq & 26 & 5 & 1 & 4 & 10 & 6 \\
\hline \multicolumn{2}{|c|}{ España } & $\begin{array}{l}\text { Totalmente en } \\
\text { desacuerdo }\end{array}$ & En desacuerdo & $\begin{array}{l}\text { Ni de acuerdo, } \\
\text { ni en desacuerdo }\end{array}$ & De acuerdo & $\begin{array}{l}\text { Totalmente de } \\
\text { acuerdo }\end{array}$ \\
\hline
\end{tabular}

9. Para aprender historia debo saber interpretar el pasado.

\begin{tabular}{c|c|c|c|c|c|c}
\hline$\%$ & 100 & 5.1 & 5.9 & 9.3 & 19.5 & 60.2 \\
\hline Frq & 118 & 6 & 7 & 11 & 23 & 71 \\
\hline
\end{tabular}


(Continuación)

\begin{tabular}{|c|c|c|c|c|c|c|}
\hline \multicolumn{2}{|c|}{ España } & $\begin{array}{c}\text { Totalmente en } \\
\text { desacuerdo }\end{array}$ & En desacuerdo & $\begin{array}{l}\text { Ni de acuerdo, } \\
\text { ni en desacuerdo }\end{array}$ & De acuerdo & $\begin{array}{c}\text { Totalmente de } \\
\text { acuerdo }\end{array}$ \\
\hline$\%$ & 100 & 10.2 & 8.5 & 14.4 & 22 & 44.9 \\
\hline Frq & 118 & 12 & 10 & 17 & 26 & 53 \\
\hline
\end{tabular}

Fuente: Elaboración propia.

En cuanto al tercer objetivo, se han escogido los ítems 18 y 19. Los resultados de los dos ítems reflejan porcentajes elevados en Portugal con un $96.1 \%$ y un $84.6 \%$ respectivamente, y con un $94.1 \%$ y un $86.4 \%$ para el alumnado español. Ambas muestras están totalmente de acuerdo en que para evaluar los contenidos históricos el examen es el principal instrumento relacionado con el aprendizaje memorístico y el conocimiento conceptual.

TABLA 3

EVALUACIÓN DE CONTENIDOS HISTÓRICOS

\begin{tabular}{|c|c|c|c|c|c|c|}
\hline \multicolumn{2}{|c|}{ Portugal } & $\begin{array}{c}\text { Totalmente en } \\
\text { desacuerdo }\end{array}$ & En desacuerdo & $\begin{array}{l}\text { Ni de acuerdo, } \\
\text { ni en desacuerdo }\end{array}$ & De acuerdo & $\begin{array}{l}\text { Totalmente de } \\
\text { acuerdo }\end{array}$ \\
\hline \multicolumn{7}{|c|}{ 18. El principal instrumento para evaluar la historia es el examen. } \\
\hline$\%$ & 100 & 0 & 0 & 0 & 3.9 & 96.1 \\
\hline Frq & 26 & 0 & 0 & 0 & 1 & 25 \\
\hline \multicolumn{2}{|c|}{ Portugal } & $\begin{array}{c}\text { Totalmente en } \\
\text { desacuerdo }\end{array}$ & En desacuerdo & $\begin{array}{l}\text { Ni de acuerdo, } \\
\text { ni en desacuerdo }\end{array}$ & De acuerdo & $\begin{array}{l}\text { Totalmente de } \\
\text { acuerdo }\end{array}$ \\
\hline
\end{tabular}

19. Para evaluar la historia se utilizan instrumentos conceptuales y memorísticos.

\begin{tabular}{c|c|c|c|c|c|c}
\hline$\%$ & 100 & 0 & 0 & 11.5 & 3.9 & 84.6 \\
\hline Frq & 26 & 0 & 0 & 3 & 22 & 1 \\
\hline \multicolumn{2}{c|}{ España } & $\begin{array}{c}\text { Totalmente en } \\
\text { desacuerdo }\end{array}$ & En desacuerdo & $\begin{array}{c}\text { Ni de acuerdo, } \\
\text { ni en desacuerdo }\end{array}$ & $\begin{array}{r}\text { De acuerdo } \\
\text { acuerdo }\end{array}$ \\
\hline
\end{tabular}

18. El principal instrumento para evaluar la historia es el examen.

\begin{tabular}{|c|c|c|c|c|c|c|}
\hline$\%$ & 100 & 0 & 0 & 1.7 & 4.2 & 94.1 \\
\hline Frq & 118 & 0 & 0 & 2 & 5 & 111 \\
\hline \multicolumn{2}{|c|}{ España } & $\begin{array}{c}\text { Totalmente en } \\
\text { desacuerdo }\end{array}$ & En desacuerdo & $\begin{array}{l}\text { Ni de acuerdo, } \\
\text { ni en desacuerdo }\end{array}$ & De acuerdo & $\begin{array}{c}\text { Totalmente de } \\
\text { acuerdo }\end{array}$ \\
\hline
\end{tabular}

19. Para evaluar la historia se utilizan instrumentos conceptuales y memorísticos.

\begin{tabular}{c|c|c|c|c|c|c}
\hline$\%$ & 100 & 0 & 5.1 & .9 & 86.4 & 7.6 \\
\hline Frq & 118 & 0 & 6 & 1 & 9 & 102 \\
\hline
\end{tabular}

Fuente: Elaboración propia.

Con respecto al cuarto objetivo, hemos seleccionado los ítems 4 y 7 dedicados a los recursos didácticos más adecuados para enseñar historia. Mientras que el alumnado portugués con un $76.9 \%$ y un $80.7 \%$ piensa que para enseñar historia es ventajoso realizar mapas históricos, comentarios de textos, trabajos de investigación y la utilización de las TIC, los alumnos españoles elevan estos porcentajes con un $83 \%$ y un $86.5 \%$ respectivamente. 
TABLA 4

RECURSOS DIDÁCTICOS PARA ENSEÑAR HISTORIA

\begin{tabular}{l|c|c|c|c|c}
\hline Portugal & $\begin{array}{c}\text { Totalmente en } \\
\text { desacuerdo }\end{array}$ & En desacuerdo & $\begin{array}{r}\text { Ni de acuerdo, } \\
\text { ni en desacuerdo }\end{array}$ & $\begin{array}{c}\text { De acuerdo } \\
\text { acuerdo }\end{array}$ \\
\hline
\end{tabular}

4. En la enseñanza de la historia es útil realizar trabajos de investigación, utilizar mapas históricos, comentarios de texto.

\begin{tabular}{|c|c|c|c|c|c|c|}
\hline$\%$ & 100 & 0 & 0 & 7.7 & 15.4 & 76.9 \\
\hline Frq & 26 & 0 & 0 & 2 & 4 & 20 \\
\hline \multicolumn{2}{|c|}{ Portugal } & $\begin{array}{c}\text { Totalmente en } \\
\text { desacuerdo }\end{array}$ & En desacuerdo & $\begin{array}{l}\text { Ni de acuerdo, } \\
\text { ni en desacuerdo }\end{array}$ & De acuerdo & $\begin{array}{c}\text { Totalmente de } \\
\text { acuerdo }\end{array}$ \\
\hline
\end{tabular}

7. La utilización de las TIC son recursos que favorecen el aprendizaje de la historia.

\begin{tabular}{c|c|c|c|c|c|c}
\hline$\%$ & 100 & 0 & 3.9 & 11.5 & 3.9 & 80.7 \\
\hline Frq & 26 & 0 & 1 & 3 & 21 & 1 \\
\hline \multicolumn{2}{c|}{ España } & $\begin{array}{c}\text { Totalmente en } \\
\text { desacuerdo }\end{array}$ & En desacuerdo & $\begin{array}{c}\text { Ni de acuerdo, } \\
\text { ni en desacuerdo }\end{array}$ & $\begin{array}{c}\text { De acuerdo } \\
\text { Detalmente de } \\
\text { acuerdo }\end{array}$ \\
\hline
\end{tabular}

4. En la enseñanza de la historia es útil realizar trabajos de investigación, utilizar mapas históricos, comentarios de texto.

\begin{tabular}{c|c|c|c|c|c|c}
\hline$\%$ & 100 & 0 & .9 & 4.2 & 11.9 & 83 \\
\hline Frq & 118 & 0 & 1 & 5 & 14 & 98 \\
\hline \multicolumn{2}{c|}{ España } & $\begin{array}{c}\text { Totalmente en } \\
\text { desacuerdo }\end{array}$ & En desacuerdo & $\begin{array}{c}\text { Ni de acuerdo, } \\
\text { ni en desacuerdo }\end{array}$ & $\begin{array}{c}\text { De acuerdo } \\
\text { Tetalmente de } \\
\text { acuerdo }\end{array}$ \\
\hline
\end{tabular}

7. La utilización de las TIC son recursos que favorecen el aprendizaje de la historia.

\begin{tabular}{c|c|c|c|c|c|c}
\hline$\%$ & 100 & 2.5 & 0 & 5.9 & 5.1 & 86.5 \\
\hline Frq & 118 & 3 & 0 & 7 & 6 & 102 \\
\hline
\end{tabular}

Fuente: Elaboración propia.

\section{GRÁFICA 1}

COMPARATIVA PORCENTUAL DEL LOGRO DE OBJETIVOS DEL ESTUDIO E ÍTEMS PARA LA MUESTRA DE ALUMNADO PORTUGUÉS

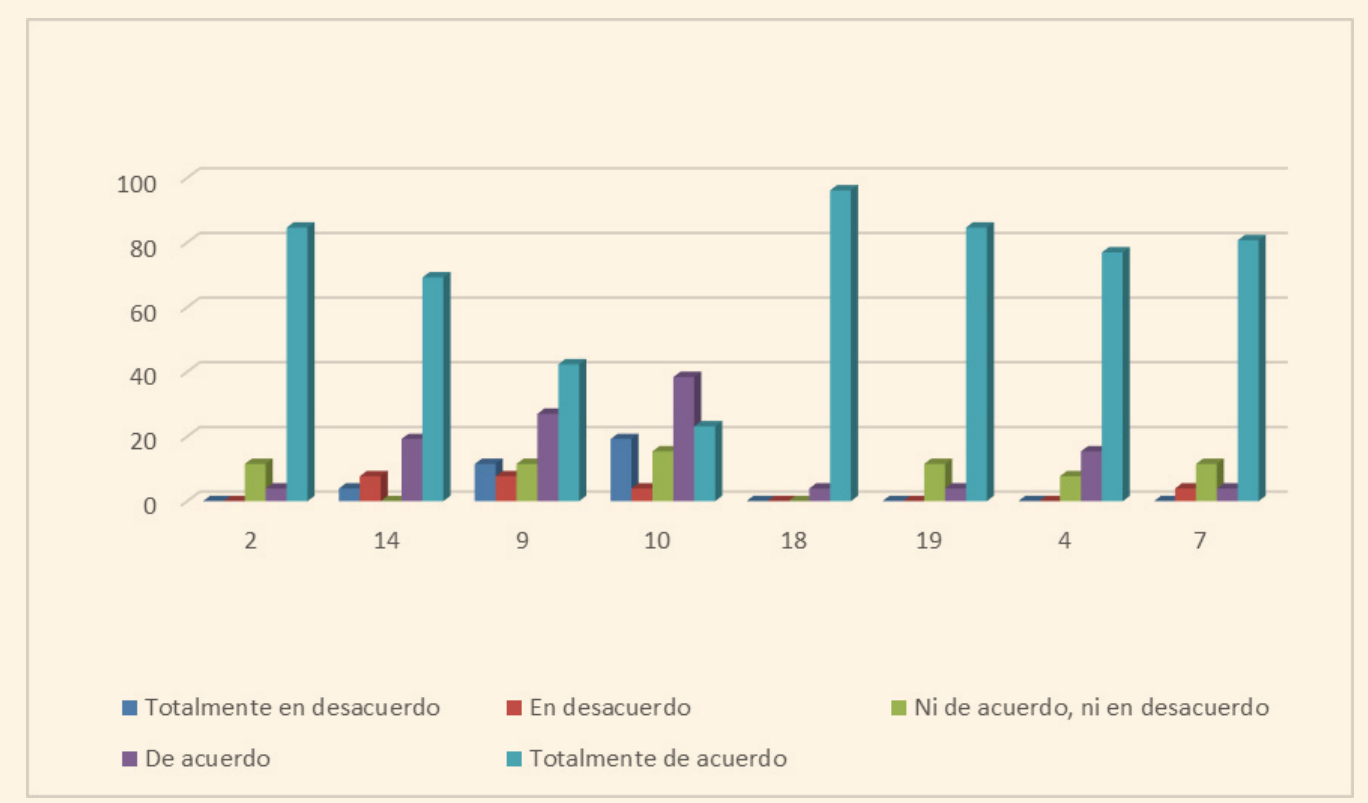

Fuente: Elaboración propia. 


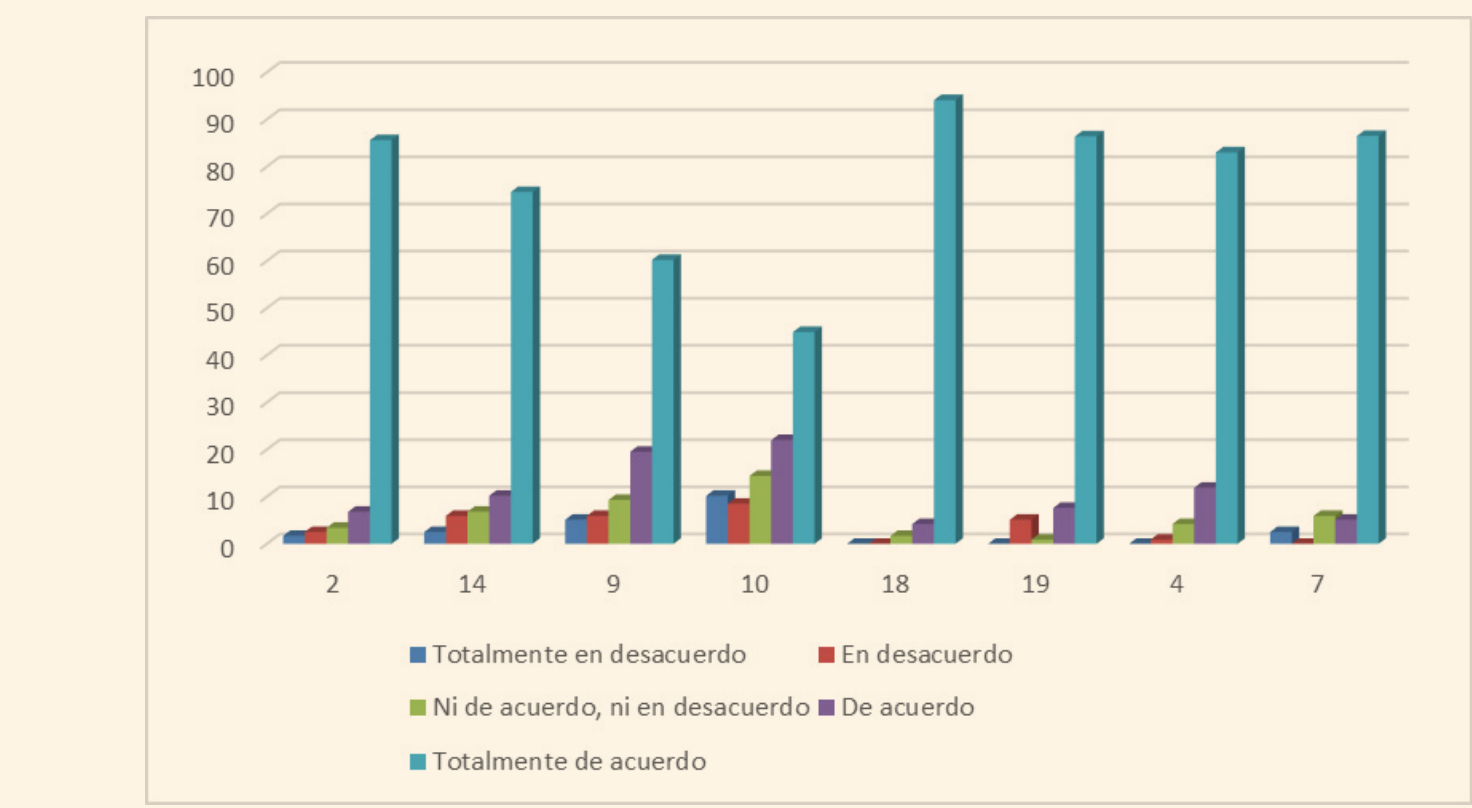

Fuente: Elaboración propia.

\section{Discusión}

Los resultados del estudio aportan de forma clara que las creencias del alumnado de España y de Portugal juegan un papel concluyente para detectar las carencias metodológicas y el desconocimiento de los métodos históricos que pueden emplear en sus clases. En esta línea, las limitaciones del estudio reflejan que el aprendizaje que adquieren en sus estudios universitarios tiene un trasfondo metodológico de corte tradicional que les hace acostumbrarse a trabajar de manera transmisiva para aprender historia mediante el uso de procedimientos conceptuales. Por tanto, es evidente que para que haya un cambio representativo de este enfoque metodológico se debe acompañar de estrategias que favorezcan la búsqueda de soluciones a los problemas que se vivencian en el mundo real y la aplicación de métodos de indagación (Gómez Carrasco et al., 2018).

A tenor de los resultados obtenidos es complicado analizar los acontecimientos del presente y el pasado y establecer diferencias o semejanzas que hagan de la historia una disciplina viva e interesante para el alumnado. Recordemos que en torno al $85 \%$ el alumnado portugués y español respondió que la metodología tradicional y expositiva protagoniza la enseñanza de la historia en la universidad. Mientras que el $70 \%$, aproximadamente, piensa que una metodología activa y participativa favorece un aprendizaje significativo. Por tanto, debemos pensar que los métodos de enseñanza que se implementan hoy día continúan primando en el relato del docente, el dictado de apuntes y el examen teórico. Aun así, están de acuerdo en realizar investigaciones, utilizar recursos digitales y también comentarios de texto para interpretar las fuentes del pasado.

Ahora bien, las concepciones de los futuros docentes de secundaria tienen un aspecto positivo y en común: sus respuestas afirmativas en los ítems relacionados con la educación histórica y el impacto práctico que tiene en su formación profesional como docentes de historia. Como indican Molina Puche et al. (2017), la historia se convierte en un instrumento para transformar la realidad y preparar al alumnado como un ciudadano crítico. No obstante, los porcentajes relacionados con el uso de fuentes directas e indirectas para facilitar el conocimiento del pasado apenas alcanzan un 23\% para Portugal y 
45\% para España. Lo que indica que quizás tengan dudas sobre la función y posibilidades de aprendizaje de las fuentes y de la interpretación del pasado a través de éstas.

En lo que respecta a la evaluación de contenidos históricos, podemos observar que no existe una cohesión de los procesos evaluativos desde el punto de vista de los métodos tradicionales o innovadores. En este ámbito, es posible encontrar estudios que exponen las oportunidades que ofrece la preparación docente en los tipos y funciones de la evaluación (König et al., 2017). Los datos de la investigación son claros al mostrar que ambos grupos están de acuerdo, con cerca de un 95\%, en que el instrumento para evaluar los conocimientos históricos es una prueba escrita, conceptual y memorística. Por último, los datos para el manejo de recursos didácticos en la enseñanza de la historia oscilan entre el 76-86\%, siendo el uso de las herramientas digitales el que tiene mejor aceptación para trabajar por competencias educativas (Miralles Martinez et al., 2019).

\section{Conclusiones}

Pese a los resultados de este estudio, la educación histórica y metodológica del alumnado de ambas universidades aporta ciertas dudas acerca de las prácticas educativas que pueden reproducir en sus clases de secundaria. Los enfoques metodológicos que han adquirido durante su aprendizaje universitario favorecen o dificultan la adquisición de competencias que son imprescindibles para elaborar propuestas y reflexionar sobre los métodos didácticos que les enriquezcan como docentes (Epstein \& Salinas, 2018). En este sentido, las problemáticas del alumnado para afrontar una metodología más activa en su desarrollo profesional dependen no solamente de la formación del docente universitario, sino de la prioridad que se da a los contenidos conceptuales frente al trabajo competencial (Muñoz et al., 2019). De esta manera, una adecuada formación inicial del profesorado novel se beneficiaría de propuestas de mejora y el diseño de actuaciones dirigidas a abordar, desde una perspectiva histórica, las causas y las consecuencias que acontecen en el desarrollo de los hechos históricos.

En esta dirección, el reto para pensar históricamente reside en formar futuros historiadores en el ámbito universitario en etapas educativas como la Educación Primaria y Secundaria, y en adquirir los beneficios de una formación histórica en la educación ciudadana (Gómez Carrasco et al., 2014). De hecho, desde la escuela a la universidad la formación del alumnado adquiere unas pautas fijas de transmisión teórica que hace de la historia una disciplina memorística y conceptual. Esta formación universitaria no ahonda en los aspectos más relevantes para su futuro desempeño docente, ni tampoco en valorar las competencias necesarias para abordar problemas de aula reales (Gómez \& Rodríguez, 2014).

Teniendo en cuenta el trabajo de León y Molina (2017), la memorización de hechos, fechas y conceptos tiene poco sentido en una sociedad donde la información está disponible en cualquier momento. Pinto y Pereira (2018) estudiaron una muestra de 130 docentes que participaron en un curso de formación continua sobre competencias digitales para dar clase en centros públicos de Educación Básica hasta Educación Secundaria de Portugal. Entre las expectativas de esta experiencia formativa se discuten las ventajas de la alfabetización digital y los principios metodológicos implementados. Esta integración de las TIC también fue analizada con las respuestas a un cuestionario de 192 estudiantes del Máster de Educación Secundaria, Bachillerato, Enseñanzas Oficiales de Idiomas y Formación Profesional y el Grado de Maestro de Infantil y Primaria. Se pudo constatar que la formación didáctica en el uso de nuevas tecnologías tuvo un efecto positivo (Roblizo et al., 2016).

Pese a la formación en competencias digitales y el uso de éstas en los centros educativos, la enseñanza de la historia continúa adscrita al modelo expositivo de aprendizaje y a la prueba escrita para evaluar contenidos conceptuales. Esto plantea futuras líneas de investigación que permanecen abiertas en la actualidad y orientan un aprendizaje enfocado en la implementación de metodologías activas de aprendizaje en la enseñanza universitaria. Por ello y para ello, la formación del profesorado en 
educación histórica es necesaria para mejorar los métodos de enseñanza de una práctica tradicional a una más innovadora, además de reforzar la formación inicial y continua de los estudiantes en competencias históricas.

\section{Agradecimientos}

Esta investigación se ha podido llevar a cabo gracias a una estancia de movilidad en el extranjero "José Castillejo", disfrutada en la Facultad de Letras de la Universidad de Oporto (Portugal) y financiada por el Ministerio de Ciencia, Innovación y Universidades de España. Asimismo, este trabajo se incluye en el grupo de investigación "Educação e Desafios Societais" del CITCEM de la Universidad de Oporto y también en el proyecto de investigación PGC2018-097481-B-I00 del Gobierno de España.

\section{Referencias}

Barca, I. (2010). Narrativas e consciência histórica dos jovens. Enseñanza de las Ciencias Sociales, (10), 23-29.

Blömeke, S., Busse, A., Kaiser, G., König, J., \& Sühl, U. (2016). The relation between content-specific and general teacher knowledge and skills. Teaching and Teacher Education, 56, 35-46. https://doi. org/10.1016/j.tate.2016.02.003

Chapman, A. (2011). Historical interpretations. In I. Davies, Debates in History Teaching (pp. 96-109). Routledge.

Domínguez, J. (2015). Pensamiento histórico y evaluación de competencias. Graó.

Epstein, T., \& Salinas, C. (2018). Research methodologies in history education. In S. A. Metzger \& L. M. Harris (Eds.), The Wiley international handbook of history teaching and learning (pp. 61-91). Wiley. https://doi.org/10.1002/9781119100812.ch3

Estepa, J., \& Martín, M. (2018). Competencia en conciencia y expresiones culturales y educación histórica patrimonios en conflicto y pensamiento crítico. In P. Miralles Martinez \& C. J. Gómez Carrasco (Eds.), La educación histórica ante el reto de las competencias: Métodos, recursos y enfoques de enseñanza (pp. 75-86). Octaedro.

Gairín, J. (2011). Formación de profesores basada en competencias. Bordón: Revista de Pedagogía, 63(1), 93-108.

García, E., \& Cabero, J. (2011). Diseño y validación de un cuestionario dirigido a describir la evaluación en procesos de educación a distancia. Edutec, 35, 1-26.

Gómez Carrasco, C. J., \& Miralles Martínez, P. (2015). ¿Pensar históricamente o memorizar el pasado? La evaluación de los contenidos históricos en la educación obligatoria en España. Revista de Estudios Sociales, (52), 52-48. https://doi.org/10.7440/res52.2015.04

Gómez Carrasco, C. J., Ortuño Molina, J., \& Miralles Martínez, P. (2018). Enseñarciencias sociales con métodos activos de aprendizaje: Reflexiones y propuestas a través de la indagación. Octaedro.

Gómez Carrasco, C. J., Ortuño Molina, J., \& Molina Puche, S. (2014). Aprender a pensar históricamente: Retos para la historia en el siglo XXI. Tempo e Argumento, 6(11), 5-27. https://doi. org/10.5965/2175180306112014005

Gómez Carrasco, C. J., \& Rodríguez Pérez, R. A. (2014). Aprender a enseñar ciencias sociales con métodos de indagación: Los estudios de caso en la formación del profesorado. REDU: Revista de Docencia Universitaria, 12(2), 307-325.

Hernández Amoros, M. J., \& Carrasco Embuena, V. (2012). Percepciones de los estudiantes del máster en formación del profesorado de educación secundaria: Fortalezas y debilidades del nuevo modelo formativo. Enseñanza \& Teaching, 30(2), 127-152.

Hernández-Pina, F., \& Maquilón, J. J. (2010). Introducción a los diseños de investigación educativa. In S. Nieto Martin (Ed.), Principios, métodos y técnicas esenciales para la investigación educativa (pp. 109-126). Dykinson. 
Ibáñez-Etxeberria, A., Gillate, I., \& Madariaga, J. M. (2015). Utilización de la historia oral para el aprendizaje de contenidos históricos en educación secundaria y su relación con la identidad local, la motivación y el autoconcepto social. Tempo e Argumento, 7(16), 204-229. https://doi. org $/ 10.5965 / 2175180307162015204$

Imbernón, F. (2019). La formación del profesorado de educación secundaria: La eterna pesadilla. Profesorado: Revista de Curriculum y Formación del Profesorado, 23(3), 151-163. https://doi.org/10.30827/ profesorado.v23i3.9302

Imbernón, F., Shigunov, A., \& Coelho, A. (2020). Reflexões sobre o conhecimento na formação de professores em comunidade de prática. Revista Iberoamericana de Educación, 82(1), 161-172. https://doi. org/10.35362/rie8213663

Jiménez, D., González, J.J., \& Tornel, M. (2018). Formación del profesorado universitario. Estudios Pedagógicos, 44(3), 157-172. http://dx.doi.org/10.4067/S0718-07052018000300157

König, J., Ligtvoet, R., Klemenz, S., \& Rothlandb, M. (2017). Effects of opportunities to learn in teacher preparation on future teachers' general pedagogical knowledge: Analyzing program characteristics and outcomes. Studies in Educational Evaluation, 53, 122-133. https://doi.org/10.1016/j. stueduc.2017.03.001

Krichesky, G. J., \& Murillo F. J. (2018). La colaboración docente como factor de aprendizaje y promotor de mejora: Un estudio de casos. Educación XX1, 21(1), 135-156. https://doi.org/10.5944/educxx1.20181

Kvale, S. (2011) Las entrevistas en investigación cualitativa. Morata.

León, E., \& Molina, M. P. (2017). Qué vienen los franceses: Una propuesta didáctica en clase de geografía e historia. In P. Sanz, J. Molero, \& D. Rodríguez. (Eds.), La Historia en el aula: I Jornadas de innovación docente y enseñanza de la Historia en la Educación Secundaria (pp. 349-360). Milenio.

Levstik, L. S. (2016). Working class connections: Identity and the teaching of history. In S. Molina, N. Llonch \& T. Martínez (Eds.), Identidad, ciudadanía y patrimonio: Educación bistórica para el siglo XXI (pp. 63-70). Trea.

Levy, S. (2017). How students navigate the construction of heritage narratives. Theory \& Research in Social Education, 45(2), 157-188. https://doi.org/10.1080/00933104.2016.1240636

Liu, J. H. (2013). Narratives and social memory from the perspective of social representations of history. In R. Cabecinhas \& L. Abadia (Eds.), Narratives and social memory: Theoretical and methodological approaches (pp. 11-24). Braga.

López-Facal, R. (2012). Los complementos de formación en el máster de secundaria. Iber: Didáctica de las Ciencias Sociales, Geografia e Historia, (71), 77-83.

Lorenzo, J. A., Muñoz, I. M., \& Beas, M. (2015). Modelos de formación inicial del profesorado de educación secundaria en España desde una perspectiva europea. Revista Complutense de Educación, 26(3), 741-757. https://doi.org/10.5209/rev_RCED.2015.v26.n3.44866

Marques, L. A. (2006). A História local como estratégia para o ensino da história. In Universidade do Porto. Estudos em Homenagem ao Professor Doutor José Marques (pp. 65-72). Porto.

Marques, L. A. (2016). Epistemologia e ensino da história. Revista História Hoje, 5(9), 9-30. https://doi. org/10.20949/rhhj.v5i9.229

Marques, L. A., \& Pinto, C. A. (2018). Formação de professores nas faculdades de letras: O exemplo de história da Universidade do Porto. In L. A. Marques (Coord.), Cruzar fronteiras sobre o ensino de história: II Oficinas Luso-Afro-Brasileiras (pp. 11-42). CITCEM.

Martínez de la Hidalga, Z., \& Villardón-Gallego, L. (2015). La imagen del profesor de educación secundaria en la formación inicial. Profesorado: Revista de Currículum y Formación del Profesorado, 19(1), 452-467.

Meschede, N., Fiebranz, A., Möller, K., \& Steffensky, M. (2017). Teachers' professional vision, pedagogical content knowledge and beliefs: On its relation and differences between pre-service and in-service teacher. Teaching and Teacher Education, 66, 158-170. https://doi.org/10.1016/j.tate.2017.04.010 
Metzger, S. A., \& Harris, L. M. (Eds.). (2018). The Wiley international handbook of history teaching and learning. Wiley.

Miralles Martinez, P., Gómez Carrasco, C. J., Arias Gonzales, V. B., \& Fontal Merillas, O. (2019). Recursos digitales y metodología didáctica en la formación inicial de docentes de Historia. Comunicar, 27(61), 45-56. https://doi.org/10.3916/C61-2019-04

Molina Puche, A., Miralles Martinez, P., Deusdad Ayala, B., \& Alfageme Gonzalez, M. B. (2017). Enseñanza de la historia, creación e identidades y prácticas docentes. Profesorado: Revista de Curriculum y Formación del Profesorado, 21(2), 331-354.

Montanares, E., \& Llancavil, D. R. (2016). Uso de fuentes históricas en formación inicial de profesores. Magis: Revista Internacional de Investigación en Educación, 8(17), 85-98.

Moriña, A. (2017). Investigar con historias de vida: Metodología biográfico-narrativa. Narcea.

Muñoz, G. A., Rodríguez, P., \& Luque, M. (2019). La formación inicial del profesorado de educación secundaria en España: Perfil y motivaciones del futuro docente. Educación XX1, 22(1), 71-92. https://doi.org/10.5944/educxx1.20007

Nóvoa, A. (2009). Para una formación de profesores construida dentro de la profesión. Revista de Educación, $350(4), 203-218$.

Pinto, M., \& Pereira, S. (2018). Experiências, perceções e expectativas da formação de professores em educação para os media em Portugal. Revista Interuniversitaria de Formación del Profesorado, 91, 83-103.

Prats, J., \& Santacana, J. (2011). ¿Por qué y para qué enseñar historia? In J. Prats (Coord.), Didáctica de la geografía y la historia (pp. 13-29). Graó.

Rivero, M. P., \& Souto, X. M. (2019). El máster de profesorado de educación secundaria: Una visión desde la especialidad de geografía e historia. Iber: Didáctica de las Ciencias Sociales, Geografía e Historia, (97), 41-50.

Roblizo, M., Sánchez, M. C., \& Cózar, R. (2016). El reto de la competencia digital en los futuros docentes de infantil, primaria y secundaria: Los estudiantes de grado y máster de educación ante las TIC. Revista de Investigación Social, (15), 254-295.

Rüsen, J. (2005). History: Narration, interpretation, orientation. Berghahn Books.

Sáiz Serrano, J., \& Fuster Garcia, C. (2014). Memorizar historia sin aprender pensamiento histórico. La PAU de Historia de España. Investigación en la Escuela, (84), 4-57.

Sáiz Serrano, J., \& Gómez Carrasco, C. J. (2016). Investigar el pensamiento histórico y narrativo en la formación del profesorado: Fundamentos teóricos y metodológicos. Revista Electrónica Interuniversitaria de Formación del Profesorado, 19(1), 175-190.

Sáiz Serrano, J., \& López-Facal, R. (2015). Competencias y narrativas históricas: El pensamiento histórico de estudiantes y futuros profesores españoles de educación secundaria. Revista de Estudios Sociales, (52), $87-101$.

Santisteban, A. (2007). Una investigación sobre cómo se aprende a enseñar el tiempo histórico. Enseñanza de las Ciencias Sociales, (6), 19-29.

Schmidt, M. A., \& Barca, I. (Orgs.). (2009). Aprender história: Perspectivas da educação histórica. Unijui.

Seixas, P. (2011). Assessment of historical thinking. In P. Clark (Ed.). New possibilities for the past, shaping history education in Canada (pp. 139-153). UBC Press.

Seixas, P., \& Morton, T. (2013). The big six historical thinking concepts. Nelson.

Serrano, R., \& Pontes, A. (2016). El desarrollo de la identidad profesional docente en la formación inicial del profesorado de secundaria. Enseñanza \& Teaching, 34(1), 35-55.

Trigueros Cano, F., Miralles Martinez, P., Molina Saorin, J., \& Maurandi Lopes, A. (2018). Percepción del alumnado sobre la evaluación de historia en educación secundaria obligatoria: Análisis de las calificaciones esperadas y obtenidas. Arbor: Ciencia, Pensamiento y Cultura, 194(788), a 449.

Vansledright, B. A. (2014). Assessing historical thinking \& understanding: Innovate designs for new standards. Routledge. 
Disponibilidad de datos

Los datos de esta investigación están disponibles en: https://data.scielo.org/dataverse/brcp

Cómo citar este artículo

Molina-Torres, M.-P. (2021). Creencias de los estudiantes del máster de secundaria en España-Portugal. Cadernos de Pesquisa, 51, Artículo e07242. https://doi.org/10.1590/198053147242

Recibido el: 27 MARZO 2020 | Aprobado para publicación en: 21 OCTUBRE 2020 